\title{
Acupuncture, Moxibustion, and Combination Therapies for Insomnia
}

\author{
Jianguo Wen', Quancheng Kan², Yan Chen ${ }^{1,2 *}$ \\ ${ }^{1}$ Institute of Clinical Medicine, The First Affiliated Hospital of Zhengzhou University, Zhengzhou, China \\ ${ }^{2}$ Department of Pharmacy, The First Affiliated Hospital of Zhengzhou University, Zhengzhou, China \\ Email: "chenyan.abby@163.com
}

Received 26 January 2016; accepted 27 June 2016; published 30 June 2016

Copyright (C) 2016 by authors and Scientific Research Publishing Inc.

This work is licensed under the Creative Commons Attribution International License (CC BY). http://creativecommons.org/licenses/by/4.0/

(c) (i) Open Access

\begin{abstract}
Insomnia, a common sleep disorder, affects general well-being, hastens the onset of other diseases, and impairs work performance. Hypnotic medications are efficacious in the short term but have obvious side effects. Acupuncture, often used to treat insomnia in traditional Chinese medicine (TCM), is considered to be beneficial in restoring the normal sleep-wake cycle by regulating and restoring the natural flow of $q i$ (energy power). The three main TCM theories for treating insomnia by acupuncture are the tranquilization disturbance, zangfu disturbance (disequilibrium of internal organs), and imbalance of yin and yang theories. Moxibustion, another treatment for insomnia, is usually combined with acupuncture. Acupuncture and moxibustion with tuina (exercise massage), acupuncture with Chinese herbal injection, electroacupuncture, and acupuncture with medication or psychotherapy are other interventions. Some acupuncture-based methods such as needle-rolling acupuncture, auricular acupoint plaster therapy, phlebotomy, and acupoint catgutembedding therapy are used as well. Although most clinical trials have shown that acupuncture and its combination therapies are significantly effective in insomnia, the beneficial effects may have been overvalued, because of small sample size, nonstrict inclusion and exclusion criteria, flawed methodology, short follow-up, or nonstandardized evaluation. Therefore, clinical studies of high methodological quality are needed to verify the efficacy of acupuncture, moxibustion, and other combination therapies in insomnia.
\end{abstract}

\section{Keywords}

Traditional Chinese Medicine, Acupuncture, Moxibustion, Auricular Acupoint, Combination Therapy

${ }^{*}$ Corresponding author. 


\section{Introduction}

Insomnia is a common sleep disorder; its predominant features are difficulty in initiating or maintaining sleep or nonrestorative sleep [1]. Its prevalence ranges from $11.7 \%$ to $37 \%$ in European countries [2], Asia [3], and the USA [4], depending on the definition used.

Western medicine holds that insomnia is the result of imbalance between cerebral excitation and inhibition, accompanied with such symptoms as irritability and peevishness, palpitation, decreased memory, emaciation, lassitude with dull complexion, fatigue, sleepiness, decreased alertness and concentration, and mood disturbances [5] [6]. In traditional Chinese medicine (TCM), the clinical manifestations of insomnia are attributed to deficiency of both the heart and the spleen, and the symptoms are dreamy sleep, palpitation, forgetfulness, lassitude, decreased food intake, postprandial abdominal distension, dull complexion, loose stools, pale tongue proper with thin-white coating, and thread-weak pulse [7]. Regardless of the description, patients are bothered by the sleep problem, which seriously affects health, hastens onset of other diseases, and impairs work performance [8]. Therefore, the therapeutic aim is to improve the quality of sleep.

For chronic insomnia, hypnotic medications (e.g., benzodiazepine receptor agonists) and cognitive-behavioral therapy (CBT) are the first-line treatments in western medicine [9]. Hypnotic medications are efficacious in the short term but have limited long-term benefits and are associated with side effects such as residual daytime sedation, cognitive impairment, and dependence [10]. CBT has been demonstrated to be effective by randomized clinical trials (RCTs) but is not widely used because of the lack of trained therapists [11]. TCM offers other therapeutic options for insomnia. TCM today is mainly used for maintaining health, enhancing physique, and managing chronic diseases such as insomnia, hemiplegia, diabetes mellitus, and hypertension.

Acupuncture and moxibustion are used to treat insomnia based on the theory of meridians [12]. Meridians are a network of passages of energy power, qi. According to Huangdi Nei Jing (Inner Classic), insomnia is a consequence of the vicious cycle of "daytime low-spirit" and "night-time hyperarousal state” [7] [13]. Acupuncture is considered to be beneficial in restoring the normal sleep-wake cycle by regulating and restoring the natural flow of qi [14]. Several Chinese clinical trials of acupuncture and moxibustion for insomnia have shown improvements in sleep quality, daytime functioning, and quality of life [15] [16]. However, international efficacy reports are limited, and the reasons are many. TCM is quite different from western medicine and difficult to understand outside traditional Chinese history and culture. Further, much of the research in TCM is recorded in ancient Chinese. The evidence is plagued by methodological limitations, high levels of heterogeneity, and publication bias [17]-[19]. Finally, TCM research is beyond the scope of most international journals. The purpose of this review is to critically examine the efficacy of acupuncture, moxibustion, and other combination therapies as alternative treatments for insomnia.

\section{Acupuncture}

Acupuncture is just one element of TCM used to treat fatigue, nausea, insomnia, diarrhea, menstrual problems, and HIV-related peripheral neuropathy [20]. It is often used in combination with exercise massage (tuina), moxibustion, electric pulsation, auricular plaster therapy, estazolam medication, and herbal therapy. The benefit of acupuncture and Chinese herbal therapy is the ability to decrease side effects associated with western treatments. In a double-dummy, single-blind, randomized, placebo-controlled clinical trial, acupuncture improved sleep quality and daytime functioning compared with sham group and estazolam medication (effective rate: 85\% vs. 24\% and 21\%) [21]. Acupuncture relieves insomnia by inducing downregulation of hypothalamic $\gamma$-aminobutyric acid (GABA) and $\gamma$-aminobutyric A receptor $(G A B A(A) R)$ in rats and lengthens pole-climbing time [22]. It can also regulate sleep and many mental and nervous symptoms, partially through increasing NOS activity and NO content, promoting normal function of brain tissues [23].

Main and supplemental acupoints differ according to the TCM theories and patient's "constitution deficiency". For example, the main acupoint Shenmen (HT 7) is the yuan (source) point of the heart channel of HandShaoyin, which dominates diseases of the heart. Needling at Shenmen (HT 7) can soothe the heart, calm the mind, relieve stuffiness of the chest, and regulate the flow of qi. The supplemental acupoints Quchi (LI 11), Fenglong (ST 40), and Jiexi (ST 41) are applied when phlegm-heat attacks the heart [7].

During acupuncture for insomnia, the patient lies in the supine position and relaxes the body naturally. After the skin is routinely sterilized, stainless silver, filiform needles $(0.25-0.3 \mathrm{~mm}$ in diameter and $25-40 \mathrm{~mm}$ in length) (Figure 1) are inserted into main and select supplemental acupoints. The needles are obliquely moved in 


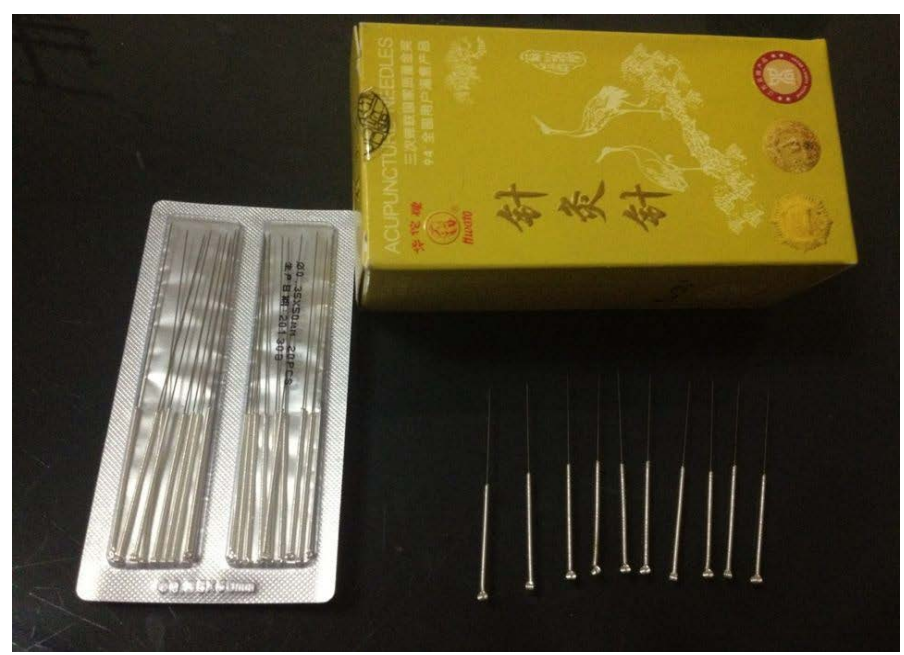

Figure 1. Filiform, stainless silver acupuncture needles $(0.25-0.3$ $\mathrm{mm}$ in diameter, 25 - $40 \mathrm{~mm}$ in length).

the forward, backward, leftward, and rightward directions to a depth of 0.5 to 1 cun $(1.7-3.3 \mathrm{~cm})$ depending on the points. The twirling reinforcing-reducing method is applied after achieving $q i$, which is essential for the therapy. The needles are retained for $30-60 \mathrm{~min}$ and manipulated twice during the session. The treatment is performed once daily for 2 - 3 courses, with 10 - 12 sessions constituting one therapeutic course and a 2-day interval between courses [15] [24]. Side effects include numbness, soreness, and turgidity, but they normalize immediately after removal of the needles. The optimal time for treatment is in the afternoon. The three relevant TCM theories are tranquilization disturbance, zangfu disturbance, and imbalance of yin and yang [7] [25] [26].

\subsection{Tranquilization Disturbance Theory}

The book Jin Yue Quan Shu, published by Zhang Jin Yue 400 years ago during the Ming dynasty, holds that insomnia results from tranquilization disturbance, so acupuncture should mainly be performed at the points for tranquilization, specifically main acupoints. Li et al. selected Baihui (DU20) and Shengting (DU24) as the main acupoints to treat 110 patients with insomnia: 67 were cured, 22 showed great improvement, 12 showed some improvement, and 9 had no change, yielding an efficacy rate of 91.81\% [27]. Cao et al. selected Baihui (DU 20), Sishenchong (EX-HN1), Taiyang (EX-HN5), Yintang (EX-HN3), Fengchi (GB20), Waiguan (SJ5), Quchi (LI11), Zushanli (ST36), Sanyinjiao (SP6), Taichong (LR3), and Qihai (RN6) as the acupoints for tranquilization and found that of 60 patients, 16 were cured, 26 showed remarkable results, 15 showed some effect and 3 were unaffected (efficacy rate of 95\%) [28].

\subsection{Zangfu Disturbance Theory (Disequilibrium of Internal Organs)}

In the chapter "Xieke" of one of the earliest TCM books, Huangdi Nei Jing-Linshu (5000 years old), it is stated that insomnia results from zangfu disturbance, so acupuncture should be mainly performed at the acupoints for zangfu. Chang et al. selected Neiguan (PC6), Shenmen (HT7), Daling (PC7), Tongli (HT5), Sanyinjiao (SP6), Baihui (DU20), and Sishenchong (EX-HN1) as the main acupoints, and applied supplemental acupoints if necessary. In their study of 40 patients, 19 were cured, 19 had a remarkable effect, 6 showed some effect, and 2 had no effect (efficacy rate of 95\%) [29].

\subsection{Imbalance of Yin and Yang Theory}

Three chapters in the book Huangdi Nei Jing·inshu, “Kouwen”, "Dahelun”, and "Genjie”, hold that qi goes mainly through the yang channel as yangqi for normal activity at daytime and the yin channel as yinqi for rest at night. When qi is still or occupies most of yang at night, the yin and yang balance is disturbed and insomnia occurs. Yang and yin are mostly separate, like positive and negative, but are not completely opposite; they are mutually blended, connected, and promoted (Figure 2). 


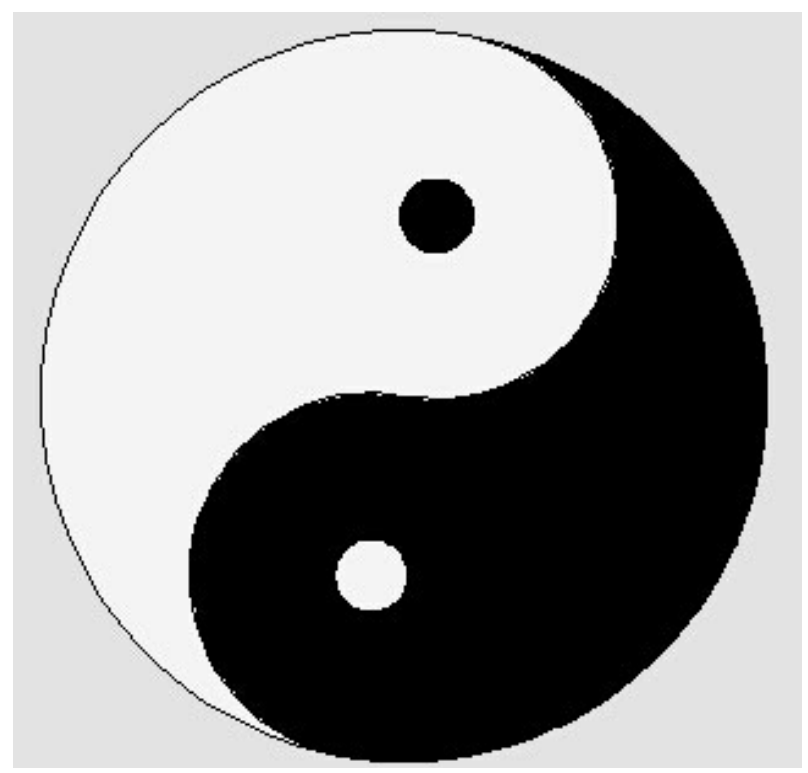

Figure 2. The yin and yang relationship of the human body. The white and black parts represent yang and yin, respectively. Yin in yang indicates some rest during the day and yang in yin means basic, vital night-time activity.

Prolonged imbalance of yin and yang would lead to disequilibrium of the whole body. The acupoints for the three types of yin and yang imbalance are as follows: 1) excess yang, Baihui (DU20), Yintang (EX-HN3), Shuigou (DU26), Zusanli (ST36), Neiguan (PC6), Sanyinjiao (SP6), Hegu (LI4), Jiuwei (CV15), Zhongting (RN16), Taichong (LR3), Neiting (ST44), and Daizhui (DU14); 2) lack of yin, Baihui (DU20), Yingtang (EX-HN3), Zusanli (ST36), Shenmen (HT7), Sanyinjiao (SP6), Taixi (KI3), Zhongting (RN16), Zhongwan (RN12), Jiuwei (RN15), Xiawan (RN10), Qihai (RV6), Guanyuan (RV4), and Daizhui (DU14); and 3) both yin and yang deficiency, Baihui (DU20), Yingtang (EX-HN3), Zusanli (ST36), Shenmen (HT7), Sanyinjiao (SP6), Hegu (LI4), Guanyuan (RN4), Dazhui (DU14), Shendao (DU11), Zhiyang (DU9), Yaoyangguan (DU3), Mingmen (DU4), and Qihai (RV6). In a study of 42 patients, Lu found that acupuncture based on this theory cured 13 patients, was remarkably effective in 10 patients, effective in 14 patients, and ineffective in 5 patients (efficacy rate of $88.1 \%)$ [30].

\section{Moxibustion}

Moxibustion is performed by transducing the heat from burning or ironing moxa or other drugs into the skin to warm the meridians, $q i$, and blood. Moxa is the most commonly used herb for moxibustion. In the treatment of insomnia, a moxa stick is mostly used and the main points are Baihui (GV 20), Zusanli (ST36), and Yongquan (KI1), according to the patient's constitution deficiency (Figure 3). Patients with "hot constitution" are not suitable for moxibustion. The best time to treat insomnia is when the patient has "cold" symptoms. An RCT of moxibustion for insomnia has shown that the treatment is as effective as estazolam medication [31], with the reported efficacy rate as high as 95\% [32].

\section{Combination Therapy}

\subsection{Acupuncture and Moxibustion}

This combination therapy is used for some serious, prolonged, or refractory cases of insomnia. Acupoints are applied according to the patient's constitution deficiency. Acupuncture is performed until $q i$ is achieved, and the needles are retained for $30 \mathrm{~min}$, during which moxibustion is performed by placing a 2-cm burning moxa stick over the needle handle. When the moxa stick is completely burnt, the ash is cleaned and the needle is withdrawn. Some physicians treated 40 patients by this combination and achieved an efficacy rate of $77.5 \%$ [33]. Another 


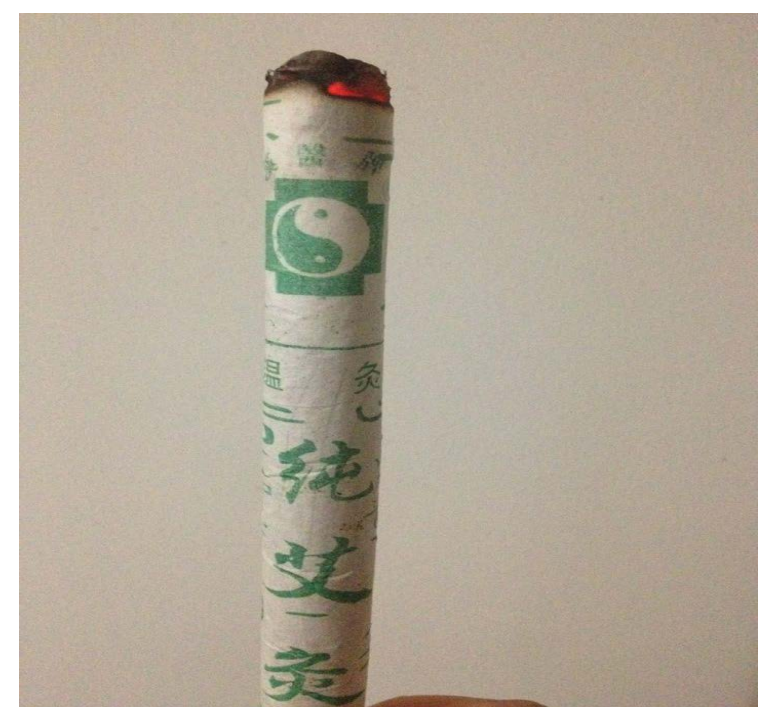

Figure 3. A lighted moxa stick for moxibustion.

research was to conduct a single-blind RCT of this combination therapy versus acupuncture alone and found that the efficacy of acupuncture with moxibustion is $87.7 \%$ and that of acupuncture only is $76.3 \%$ [34].

\subsection{Acupuncture and Moxibustion with Tuina}

Acupuncture and moxibustion performed with tuina can influence activity of the cerebral cortex and regulate nervous function to restore balance without the side effects associated with sedatives. When performed in the head region, tuina can help capillary dilatation, increase cerebral blood flow, and improve cerebral blood circulation. It is usually performed before acupuncture and moxibustion. The patient sits or lies supine and either the head or the feet, the back or the abdomen, or a combination of these parts are massaged. For tuina on the head, one-finger pushing manipulation, wiping manipulation with both thumbs, and sweeping and arching-pushing manipulations are alternately performed along the distribution of the gallbladder channel on both sides. The efficacy rate was found to be over 95\% for tuina on the head performed by five-finger grasping manipulation of the vertex to three-finger grasping manipulation of the suboccipital part in addition to acupuncture [35]. For tuina on acupoints of the feet, the reported efficacy rate is $96 \%$ [36].

\subsection{Acupuncture with Chinese Herbal Injection}

Some TCM practitioners use injectors instead of silver needles for acupuncture. After achieving qi, 1 - $3 \mathrm{ml}$ of Chinese herbs, usually gastrodine or salvianolic acid extracted from Salvia miltiorrhiza, is injected into the acupoints. This treatment is performed daily, with 10 days constituting one therapeutic course and a 3-day interval between courses. Shi et al. found that the efficacy rate after 2 courses in patients with insomnia was 95\% [37].

\subsection{Electroacupuncture}

In electroacupuncture (EA), conventional acupoints are used, but the needles are connected to the output terminals of an EA apparatus. Continuous waves at medium current frequency (e.g., $80 \mathrm{~Hz}$ ) are used. The intensity is adjusted to induce slight muscular twitching $(\sim 1-3 \mathrm{~mA})$ and lasts for $30 \mathrm{~min}$. Continuous treatment over 10 days is considered one course. Four courses are performed in each patient with an interval of 3 days. A clinical trial has demonstrated the obvious therapeutic effect of EA on insomnia [38].

\subsection{Acupuncture with Medication or Psychotherapy}

With the development of western medicine in China, TCM has been integrated with western medicine. In this field, insomnia is usually treated with a combination of herbs, acupuncture, estazolam medication, and psychotherapy, yielding an efficacy rate of over 90\% [39] [40]. 


\section{Special Acupuncture Methods}

\subsection{Needle-Rolling Acupuncture}

Needle-rolling acupuncture is an old method based on acupuncture. It gently stimulates a larger area on the channels and collaterals where the 5 zang Shu-Mu points are located, strengthening functions of the zang-fu organs. Through the channels and collaterals, this stimulation also acts on the brain, regulating yin, yang, qi, and blood to treat insomnia. The spinal nerves form branches on both sides of the spine, and in the deep layer, the sympathetic trunk, sympathetic paravertebral ganglia, and gray and white ramus communicans join the spinal nerves. Therefore, large-area stimulation by needle-rolling therapy is likely to effectively adjust disturbed function of vegetative nerves and the microcirculatory system at the body surface (in the skin) as well as activate regulatory nerves of the corresponding internal organs. It may also influence the release of chemical mediators of the peripheral and central nervous systems, such as dopamine, noradrenalin, acetylcholine, somatotropin, and corticotrophin-releasing factor, thus inducing therapeutic effects.

In this method, the patient lies in the prone position. The operator uses the needle-rolling instrument in the following order: 1) rolling along the first line of the bladder channel from Feishu (BL 13) to Shenshu (BL 23); 2) rolling along the second line of the bladder channel from Dazhu (BL 11) to Zhishi (BL 52); and 3) rolling along the governor channel from Mingmen (GV 4) to Dazhui (GV 14). Needle rolling is performed quite slowly about 10 times, with the rolling force maintained at the patient's comfort level but sufficient to redden the skin (indicating smooth flow of $q i$ and blood in the channel). Each session lasts 15 - $20 \mathrm{~min}$. In a previous study, the treatment group received needle-rolling therapy once daily in the daytime, for 15 - 20 min per session, and 5 times a week with a one-day interval [41]. An RCT of the therapeutic effect on insomnia showed an efficacy rate of $82.2 \%$ in the needle-rolling group and $60.0 \%$ in the clonazopam medication group after 4 weeks, although no significant difference was noted after the 3-month follow-up. Regardless, the needle-rolling group had better life quality due to fewer side effects [42] [43].

\subsection{Auricular Acupoint Plaster Therapy}

According to TCM, the auricular surface mainly communicates with collateral channels of the inner organs and limbs. The shape of the auricle is like an inverted fetus, so the distribution of functional acupoints is similar to that of inverted fetal organs. In general, the auricular acupoints of the head and face are located at the earlobe, those of the upper limb are on the scaphoid, those of the trunk and lower limb are located on the helix and its foot, and the acupoints of the visceral organs are in the concha and its cavity (Figure 4).

Auricular acupoints are applied to treat many diseases in several methods (e.g., acupuncture, moxibustion, phlebotomy, herbal plaster therapy, or their combinations). Auricular acupoint plaster therapy is most commonly used for insomnia, and the main auricular acupoints are ear-Shenmen, heart, mouth (also called "sleep-promoting point"), occiput, anterior lobe of forehead (also called "neurasthenia point"), and subcortex of forehead. Supplemental points are selected as follows: spleen for deficiency of the heart and spleen, kidney for disharmony between the heart and the kidney, liver for upward disturbance of liver-fire, gallbladder for deficiency of heart-qi and gallbladder-qi, and stomach for dysfunction of the stomach [44] [45]. Liu treated 40 cases and found the efficacy rate to be $90 \%$ [46].

\subsection{Phlebotomy}

Phlebotomy (bloodletting) has a long history and has been widely applied. To treat insomnia, acupoints on the ear are selected according to the patient's constitution deficiency. The ear is sterilized, a needle is used to prick the selected acupoint, the earlobe is gently squeezed to release 4 - 5 drops of blood, and the punctured point is pressed with a clean swab to stop bleeding. This procedure can be repeated twice a week, on alternate sides each time. Contraindications include bleeding disorders, anemia, hypotension, and pregnancy. Although phlebotomy is extensively performed in China, the method needs further research and summarization [47] [48].

\subsection{Acupoint Catgut-Embedding Therapy}

Acupoint catgut-embedding therapy integrates a variety of treatments including acupuncture, needle embedding, and tissue therapy. The method is based on acupuncture and involves implantation of an absorbable thread-like 


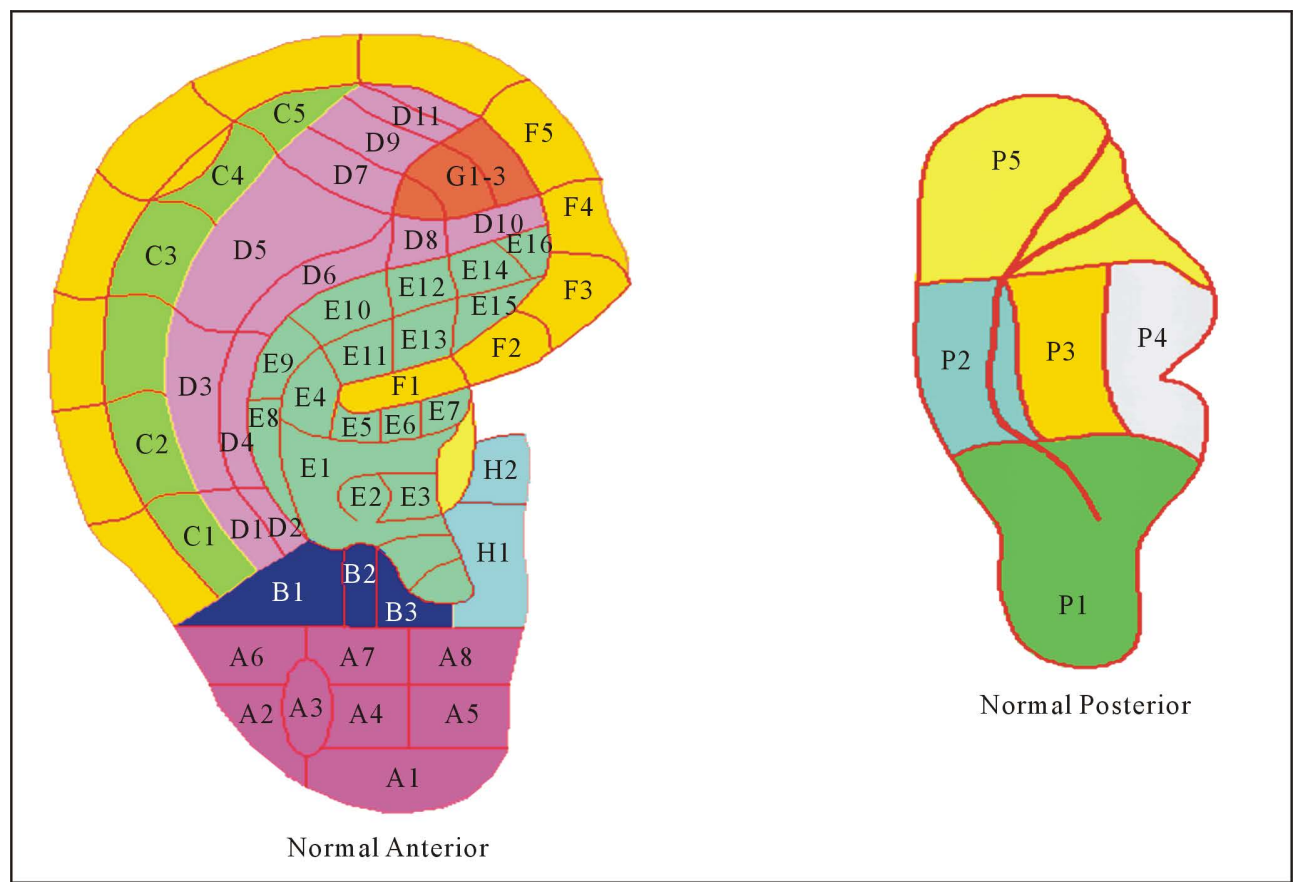

Figure 4. The auricular acupoint graph. Area A (earlobe): A1, tonsil; A2, inner ear; A3, cheek; A4, eye; A5, vertical front; A6, jaw; A7, tongue; A8, teeth. Area B: B1, occiput; B2, temple; B3, forehead. Area C (periotic auriculae): C1, clavicle; C2, elbow; C3, shoulder; C4, wrist; C5, finger. Area D (antihelix): D1, cervical vertebra; D2, neck; D3, thoracic vertebra; D4, thorax; D5, lumbosacral vertebrae; D6, abdomen; D7, hip; D8, buttocks; D9, knee; D10, sciatic nerve; D11, ankle. Area E (concha auriculae): E1, lung; E2, heart; E3, trachea; E4, stomach; E5, cardia; E6, esophagus; E7, mouth; E8, spleen; E9, liver; E10, pancreas and gallbladder; E11, duodenum; E12, kidney; E13, small intestine; E14, bladder; E15, colon; E16, sacrum. Area F (helix): F1, middle ear; F2, rectum; F3, urethra; F4, external genital organs; F5, anus. Areas G1-3 (fossa triangularis auriculae): internal genitalia. Area $\mathrm{H}$ (tragus): H1, external nose; H2, throat and inner nose. Posterior auricular acupoints: P1, kidney; P2, liver; P3, spleen; P4, lung; P5, heart.

protein, such as catgut, into 3 - 5 corresponding line-standing acupoints. Both acupuncture and catgut-embedding therapy can improve sleep quality, but catgut-embedding therapy is less time consuming and more convenient, and ensures longer stimulation [49] [50].

\section{Conclusion}

Most clinical trials of insomnia have shown that, compared with no treatment, sham acupuncture, or medication, acupuncture significantly improves sleep quality and duration, and the efficacy rate varies from 70\% to 98\% [7] [25] [26]. The combination of acupuncture and other TCM interventions appears to be more effective than individual interventions. The beneficial effects of acupuncture may have been overvalued in some trials, because of small sample size, nonstrict inclusion and exclusion, flawed methodology, and short follow-up. Moreover, conventional tools such as the Pittsburgh Sleep Quality Index, Athens Insomnia Scale, Insomnia Severity Index, and Epworth Sleepiness Scale were seldom used to evaluate the results. These scales have inherent problems, such as subjectivity, lack of standardization and generalizability, and limited applicability of the Life Quality Scale [50]. Clinical trials with high methodological quality, especially RCTs, are needed to validate the efficacy of acupuncture, moxibustion, and their combinations for insomnia.

\section{References}

[1] Kyle, S.D., Beattie, L., Spiegelhalder, K., Rogers, Z. and Espie, C.A. (2014) Altered Emotion Perception in Insomnia Disorder. Sleep, 7, 775-783. http://dx.doi.org/10.5665/sleep.3588

[2] Pallesen, S., Sivertsen, B., Nordhus, I.H. and Bjorvatn, B. (2014) A 10-Year Trend of Insomnia Prevalence in the 
Adult Norwegian Population. Sleep Medicine, 15, 173-179. http://dx.doi.org/10.1016/j.sleep.2013.10.009

[3] Izuhara, K., Wada, K., Nakamura, K., Tamai, Y., Tsuji, M., Ito, Y., et al. (2013) Association between Tinnitus and Sleep Disorders in the General Japanese Population. Annals of Otology, Rhinology \& Laryngology, 122, 701-706. http://dx.doi.org/10.1177/000348941312201107

[4] Calhoun, S.L., Fernandez-Mendoza, J., Vgontzas, A.N., Liao, D. and Bixler, E.O. (2014) Prevalence of Insomnia Symptoms in a General Population Sample of Young Children and Preadolescents: Gender Effects. Sleep Medicine, 15, 91-95. http://dx.doi.org/10.1016/j.sleep.2013.08.787

[5] Green, M.J., Espie, C.A. and Benzeval, M. (2014) Social Class and Gender Patterning of Insomnia Symptoms and Psychiatric Distress: A 20-Year Prospective Cohort Study. BMC Psychiatry, 14, 152. http://dx.doi.org/10.1186/1471-244X-14-152

[6] Armstrong, J.M., Ruttle, P.L., Klein, M.H., Essex, M.J. and Benca, R.M. (2014) Associations of Child Insomnia, Sleep Movement, and Their Persistence with Mental Health Symptoms in Childhood and Adolescence. Sleep, 37, 901-909. http://dx.doi.org/10.1186/1471-244X-14-152

[7] Sun, G.J. (2002) Acupuncture. People’s Medical Publishing House, Beijing, 720. [In Chinese]

[8] Léger, D., Bayon, V., Ohayon, M.M., Philip, P., Ement, P., Metlaine, A., et al. (2014) Insomnia and Accidents: CrossSectional Study (EQUINOX) on Sleep-Related Home, Work and Car Accidents in 5293 Subjects with Insomnia from 10 Countries. Journal of Sleep Research, 23, 143-152. http://dx.doi.org/10.1111/jsr.12104

[9] Cunnington, D., Junge, M.F. and Fernando, A.T. (2013) Insomnia: Prevalence, Consequences and Effective Treatment. Medical Journal of Australia, 199, S36-S40. http://dx.doi.org/10.5694/mja13.10718

[10] Pigeon, W.R., Woosley, J.A. and Lichstein, K.L. (2014) Insomnia and Hypnotic Medications Are Associated with Suicidal Ideation in a Community Population. Archives of Suicide Research, 18, 170-180. http://dx.doi.org/10.1080/13811118.2013.824837

[11] Taylor, D.J. and Pruiksma, K.E. (2014) Cognitive and Behavioural Therapy for Insomnia (CBT-I) in Psychiatric Populations: A Systematic Review. International Review of Psychiatry, 26, 205-213. http://dx.doi.org/10.3109/09540261.2014.902808

[12] Lu, D.P. and Lu, G.P. (2013) An Historical Review and Perspective on the Impact of Acupuncture on U.S. Medicine and Society. Medical Acupuncture, 25, 311-316. http://dx.doi.org/10.1089/acu.2012.0921

[13] Park, J., Linde, K., Manheimer, E., Molsberger, A., Sherman, K., Smith, C., et al. (2008) The Status and Future of Acupuncture Clinical Research. Journal of Alternative and Complementary Medicine, 14, 871-881. http://dx.doi.org/10.1089/acm.2008.SAR-4

[14] Hempel, S., Taylor, S.L., Solloway, M.R., Miake-Lye, I.M., Beroes, J.M., Shanman, R., et al. (2014) Evidence Map of Acupuncture. Department of Veterans Affairs, Washington DC. http://www.ncbi.nlm.nih.gov/pubmedhealth/PMH0063214/

[15] Zhao, K. (2013) Acupuncture for the Treatment of Insomnia. International Review of Neurobiology, 111, 217-234. http://dx.doi.org/10.1016/B978-0-12-411545-3.00011-0

[16] Tang, Y., Zhang, L.X., Hu, Y.P., Wu, J., Lu, P., Liu, M. and Luo, R. (2013) Clinical Research of Present Situation on Acupuncture Treatment for Insomnia in Recent 5 Years. Journal of Liaoning University of Traditional Chinese Medicine, 15, 143-144. (In Chinese) http://www.cnki.com.cn/Article/CJFDTotal-LZXB201307060.htm

[17] Cheuk, D.K., Yeung, W.F., Chung, K.F. and Wong, V. (2012) Acupuncture for Insomnia. Cochrane Database of Systematic Reviews, No. 9, Article ID: CD005472. http://dx.doi.org/10.1002/14651858.cd005472.pub3

[18] Cao, H., Pan, X., Li, H. and Liu, J. (2009) Acupuncture for Treatment of Insomnia: A Systematic Review of Randomized Controlled Trials. The Journal of Alternative and Complementary Medicine, 15, 1171-1186. http://dx.doi.org/10.1089/acm.2009.0041

[19] Yeung, W.F., Chung, K.F., Leung, Y.K., Zhang, S.P. and Law, A.C. (2009) Traditional Needle Acupuncture Treatment for Insomnia: A Systematic Review of Randomized Controlled Trials. Sleep Medicine, 10, 694-704. http://dx.doi.org/10.1016/j.sleep.2008.08.012

[20] Chavez, C. (1995) Prickly Business. The Finer Points of Acupuncture. Positively Aware, Jan-Feb, 14-15.

[21] Guo, J., Wang, L.P., Liu, C.Z., Zhang, J., Wang, G.L., Yi, J.H., et al. (2013) Efficacy of Acupuncture for Primary Insomnia: A Randomized Controlled Clinical Trial. Evidence-Based Complementary and Alternative Medicine, 2013, Article ID: 163850. http://dx.doi.org/10.1155/2013/163850

[22] Zhou, Y.L., Gao, X.Y., Wang, P.Y. and Ren, S. (2012) Effect of Acupuncture at Different Acupoints on Expression of Hypothalamic GABA and GABA(A) Receptor Proteins in Insomnia Rats. Acupuncture Research, 37, 302-307. (In Chinese)

[23] Gao, X.Y., Ma, Q.L. and Hu, B. (2007) Effects of Acupuncture at "Sishencong” (EX-HN 1) on Physiological Func- 
tions in the Sleep Disorder Model Mouse. Chinese Acupuncture \& Moxibustion, 27, 681-683. (In Chinese)

[24] Liu, C.Z., Litscher, G., Liang, F.R., Kong, J., Wang, L.P. and Wang, L. (2014) Deqi Sensation in Different Kinds of Acupuncture. Evidence-Based Complementary and Alternative Medicine, 2014, Article ID: 121573. http://dx.doi.org/10.1155/2014/121573

[25] Li, L.F. and Lu, J.H. (2010) Clinical Observation on Acupuncture Treatment of Intractable Insomnia. Journal of Traditional Chinese Medicine, 30, 21-22. http://dx.doi.org/10.1016/S0254-6272(10)60006-4

[26] Xia, X.J., Xie, J.G. and Zhang, S.Q. (2009) A Test on Composing Time of Huang Di Nei Jing. Gansu Journal of Traditional Chinese Medicine, 22, 4-5. (In Chinese) http://www.cqvip.com/QK/97444X/200905/30383803.html

[27] Li, Z.P. (2006) Clinical Observation on Treatment of 110 Cases of Insomnia with Acupuncture Baihui (GV 20) and Shenting (GV 24). Journal of Clinical Acupuncture and Moxibustion, 22, 38-39. (In Chinese)

[28] Cao, H., Jin, Z. and Lu, X. (2008) Clinical Observation on Treatment of 60 Cases of Insomnia with Fu Zheng An Shen Acupuncture. Jilin Journal of Traditional Chinese Medicine, No. 1, 45. (In Chinese) http://www.cqvip.com/QK/93527X/200801/26477767.html

[29] Zhou, Y., Du, C. and Gao, X. (2009) Puncture Back Shu-Acupoints to Treat 40 Cases of Insomnia. Traditional Chinese Medicinal Research, 22, 56-57. (In Chinese)

[30] Lu, J. (2008) Clinical Observation on Treatment of Insomnia with Acupuncture. Shanghai Journal of Acupuncture and Moxibustion, 27, 6-7. http://d.wanfangdata.com.cn/Periodical_shzjzz200802003.aspx

[31] Ju, Y.L., Chi, X. and Liu, J.X. (2009) Forty Cases of Insomnia Treated by Suspended Moxibustion at Baihui (GV 20). Journal of Traditional Chinese Medicine, 29, 95-96. (In Chinese) http://dx.doi.org/10.1016/S0254-6272(09)60040-6

[32] Lü, P.W. (2011) Moxibustion Yintang (EX-HN 3) Acupoint for Insomnia of Sleep Difficulty. Chinese Acupuncture \& Moxibustion, 31, 786. (In Chinese)

[33] Wu, H., Yu, A. and Yu, X. (2013) Observation on the Therapeutic Effect of Acupuncture and Moxibustion on Insomnia. Shanghai Journal of Acupuncture and Moxibustion, 32, 497-498. (In Chinese)

[34] Gao, X., Xu, C., Wang, P., Ren, S., Zhou, Y., Yang, X., et al. (2013) Curative Effect of Acupuncture and Moxibustion on Insomnia: A Randomized Clinical Trial. Journal of Traditional Chinese Medicine, 33, 428-432. http://dx.doi.org/10.1016/S0254-6272(13)60143-0

[35] Kong, F.Z. and Zhou, Q.Y. (2004) Electric Acupuncture with Tuina to Treat 86 Insomnia Patients. Journal of Clinical Acupuncture and Moxibustion, 20, 8-9. (In Chinese) http://www.cnki.com.cn/Article/CJFDTotal-ZJLC200401005.htm

[36] Yang, J.X. (2004) Acupuncture with Tuina on Feet to Treat 58 Insomnia Patients. Journal of Clinical Acupuncture and Moxibustion, 20, 9. (In Chinese) http://www.cnki.com.cn/article/cjfdtotal-zjlc200401006.htm

[37] Shi, L., Chen, J. and Zhang, J. (2010) Clinical Research of Gastrodin Acupoint Injection in the Treatment of Insomnia. Journal of External Therapy of Traditional Chinese Medicine, 19, 41-44. (In Chinese)

[38] Tang, S.C., Liu, J.M. and Liu, G.L. (2007) Clinical Observation on Effect of Electric Acupuncture at Sishencong in Treating Insomnia. Chinese Journal of Integrated Traditional and Western Medicine, 27, 1030-1032. (In Chinese)

[39] Han, Z. (2010) Clinical Observation on Treatment of Insomnia by Acupuncture and Medication. Chinese Journal of Modern Drug Application, 15, 149. (In Chinese) http://www.cnki.com.cn/Article/CJFDTotal-ZWYY201015134.htm

[40] He, J.M., Chen, C.H., Zhang, H.P., Liu, M., Ou, L.F., Tan, X.Y., et al. (2007) The Combination of Psychological Therapy and Acupuncture in the Treatment of Insomnia. Chinese Journal of Rehabilitation Theory and Practice, 13, 85. (In Chinese) http://www.cnki.com.cn/Article/CJFDTOTAL-ZKLS200701035.htm

[41] Luo, L., Hu, Y.P., Yu, S.G. and Li, N. (2006) Observation on Therapeutic Effect of Rolling Needle Therapy on Insomnia. Chinese Acupuncture \& Moxibustion, 26, 183-185. (In Chinese)

[42] Huang, L.S., Wang, D.L., Wang, C.W., Hu, Y.P., Zhou, J.W. and Li, N. (2009) The Needle-Rolling Therapy for Treatment of Non-Organic Chronic Insomnia in 90 Cases. Journal of Traditional Chinese Medicine, 29, 19-23. http://dx.doi.org/10.1016/S0254-6272(09)60025-X

[43] Wang, C.W., Kang, J., Zhou, J.W., Hu, Y.P. and Li, N. (2006) Effect of Rolling Needle Therapy on Quality of Life in the Patient of Non-Organic Chronic Insomnia: A Randomized Controlled Trial. Chinese Acupuncture \& Moxibustion, 26, 461-465. (In Chinese)

[44] Yao, S. (1999) 46 Cases of Insomnia Treated by Semiconductor Laser Irradiation on Auricular Points. Journal of Traditional Chinese Medicine, 19, 298-299.

[45] Yeung, W.F., Chung, K.F., Poon, M.M., Ho, F.Y., Zhang, S.P., Zhang, Z.J., et al. (2012) Acupressure, Reflexology, and Auricular Acupressure for Insomnia: A Systematic Review of Randomized Controlled Trials. Sleep Medicine, 13, 971-984. http://dx.doi.org/10.1016/j.sleep.2012.06.003

[46] Liu, W. (2007) Forty Cases of Insomnia Treated by Multi-Output Electric Pulsation and Auricular Plaster Therapy. 
Journal of Traditional Chinese Medicine, 27, 106-107.

[47] Xu, R. (1990) The Application of Bloodletting in Acupuncture Therapy. Journal of Traditional Chinese Medicine, 10, 274-275.

[48] Zeng, S.J. (2013) Explanation on Bloodletting Mechanism of Scraping Therapy. Chinese Acupuncture \& Moxibustion, 33, 1033-1035. (In Chinese)

[49] Li, Z.P. (2006) Fifty-Two Cases of Insomnia Treated by Acupoint Catgut-Embedding Therapy. Journal of New Chinese Medicine, 38, 68-69.

[50] Yang, Y., Wang, L.P., Zhang, L. and Guo, J. (2013) Application and Selection of Insomnia Scales in Acupuncture Clinical Research. Chinese Acupuncture \& Moxibustion, 33, 1039-1042. (In Chinese)

\section{Submit or recommend next manuscript to SCIRP and we will provide best service for you:}

Accepting pre-submission inquiries through Email, Facebook, Linkedin, Twitter, etc A wide selection of journals (inclusive of 9 subjects, more than 200 journals)

Providing a 24-hour high-quality service

User-friendly online submission system

Fair and swift peer-review system

Efficient typesetting and proofreading procedure

Display of the result of downloads and visits, as well as the number of cited articles

Maximum dissemination of your research work

Submit your manuscript at: http://papersubmission.scirp.org/ 